symptomatic before 5 years of age. The CMT typical phenotype in some was complicated by delayed motor development, sensorineural hearing loss, tremor, and pathological fractures. Axonal loss affected all patients. An X-linked dominant inheritance and carrier females with abnormal exam correlated with a connexin 32 mutation in all but 2 pedigrees. The clinical phenotype for CMTX1 is broader than previously recorded. (Yiu EM et al. Neurology Feb 2011;76:461-466).

\title{
SERUM TRANSAMINASE IN DUCHENNE DYSTROPHY
}

Researchers at Children's Hospital Boston, MA, have shown a linear relationship between serum CPK and serum ALT and AST and a logarithmic relationship between serum enzyme levels and age for boys with Duchenne $(n=46)$ or Becker $(n=9)$ muscular dystrophy (DMD or BMD). A mathematical model to predict serum ALT and AST levels with known CPK and age was developed to provide reassurance that elevated transaminase may be indicative of muscle disease, avoiding unnecessary tests of liver dysfunction. Serum transaminase was highest in ambulant boys with DMD (1220 U/L ALT and $801 \mathrm{U} / \mathrm{L}$ AST). These levels were 22 and 12 times higher than upper limit nomal levels for ALT and AST, respectively. The study was prompted by the observed reluctance of clinicians to attribute high transaminase levels to muscle disease, leading to delayed diagnosis and extensive tests for liver dysfunction. (McMillan HJ, Gregas M, Darras BT, Kang PB. Serum transaminase levels in boys with Duchenne and Becker muscular dystrophy. Pediatrics Jan 2011;127:e132-e136). (Respond: Peter B Kang MD, Department of Neurology, Children's Hospital Boston, 300 Longwood Ave, Boston, MA 02115. E-mail: peter.kang@ childrens.harvard.edu).

COMMENT. High serum transaminase levels should alert clinicians to possible muscle disease and prompt serum CPK estimation when clinically indicated. Unnecessary liver function tests and withdrawal of drug therapy may be avoided.

\section{MRI DEFINITION OF INVOLVED MUSCLE IN DUCHENNE MUSCULAR DYSTROPHY}

The degree of muscle involvement of lower leg muscles of 34 patients with DMD $>8$ years, using muscle MRI, was estimated in a multicenter study at the Institute of Child Health and other centers in London, in the UK, and 1 in Rome, Italy. Muscle MRI findings in a subgroup of 15 patients were correlated with the histology of open biopsies of extensor digitorum brevis (EDB). A gradient of muscle involvement in the lower leg was documented in all patients, and the posterior compartment (gastrocnemius $>$ soleus) was most severely affected. The anterior compartment (tibialis anterior/posterior, popliteus, extensor digitorum) was least affected. Muscle MRI/EDB involvement correlated with the patient's age $(\mathrm{p}=0.055)$. MRI correlated with EDB histopathologic changes in 10/15 patients. Abnormal MRI grades 3-4 (range 0-4) were associated with more severe fibro-adipose tissue replacement. Muscle MRI showed a progressive involvement of the EDB, more obvious in older patients and those nonambulant for a longer time. (Kinali V, Arechavala-Gomeza V, Cirak S, et al. Muscle histology vs MRI in Duchenne muscular dystrophy. Neurology Jan 25, 2011;76:346-353). (Respond: Dr 
Francesco Muntoni, The Dubowitz Neuromuscular Centre, First Floor, UCL, Institute of Child Health, 30 Guildford Street, London, UK. E-mail: $\underline{\text { f.muntoni@ich.ucl.ac.uk). }}$

COMMENT. This study demonstrates good correlation between muscle MRI and muscle histologic changes in boys with DMD. It validates muscle MRI as a tool for selection of patients with sufficiently preserved EDB muscle for clinical trials of IM injections of an antisense oligonucleotide $(\mathrm{AO})$ to induce dystrophin production.

\section{VASCULAR DISORDERS}

\section{ARTERIAL ISCHEMIC STROKE STUDY OF RISK FACTORS}

Data from the International Pediatric Stroke Study were analyzed by researchers at Royal Children's Hospital, Melbourne, Australia, and other centers. Risk factors (RFs) for arterial ischemic stroke (AIS) and their characteristics were identified in a total of 676 patients. RFs included arteriopathies (53\%), cardiac disorders (31\%), infection (24\%), acute head and neck disorders $(23 \%)$, acute systemic disorders $(22 \%)$, chronic systemic conditions $(19 \%)$, prothrombotic states $(13 \%)$, chronic head and neck disorders (10\%), atherosclerosis-related RFs (2\%), other (22\%), and none was identified in $9 \%$. RFs were multiple in $52 \%$ patients. Prevalence of RFs varied with geography and with age: arteriopathy was relatively less prevalent in Asia and prothrombotic states more prevalent in Europe; cardiac disease was most prevalent in preschoolers, arteriopathies in children 5 to 9 years old, and chronic head and neck disorders were highest in children aged 10 to 14 years. Arteriopathies were associated with focal signs and multiple infarcts, and cardiac disease with hemorrhagic conversion. (Mackay MT, Wiznitzer M, Benedict SL, Lee KJ, deVeber GA, Ganesan V. Arterial ischemic stroke risk factors: the International Pediatric Stroke Study. Ann Neurol Jan 2011;69:130-140). (Respond: Dr Mark T Mackay, Children's Neuroscience Centre, Royal Children's Hospital, Flemington Rd, Parkville, Victoria, Australia 3052. E.mail: mark.mackay@rch.org.au).

COMMENT. RFs, especially arteriopathy and cardiac disorders, are common in childhood AIS. Emphasis of investigation and preventive therapies may be determined by geographical and age-related prevalences of risk factors.

\section{OUTCOME OF CEREBELLAR HEMORRHAGE IN PRETERM NEWBORNS}

Long-term neurodevelopmental outcome of preterm infants with cerebellar hemorrhages detected only on MRI was studied at University of California, San Francisco. Of 131 preterm newborns evaluated by cranial ultrasound and MRI, cerebellar hemorrhage was seen on ultrasound in 3 and confirmed on MRI. An additional 10 cerebellar hemorrhages not detected by ultrasound were seen on MRI. Three newborns died in the nursery, 2 with ultrasound-detected cerebellar hemorrhages. Of 128 survivors, 94 underwent periodic neurodevelopmental exams until age 3-6 years (mean 4.8). Of 8 newborns with cerebellar hemorrhage seen only on MRI and assessed at age 3-6 years, 